\title{
Classical Bounds on Quantum Partition Functions`
}

\author{
Bernhard Baumgartner \\ Institut für Theoretische Physik, Universität Wien, A-1090 Wien, Austria
}

\begin{abstract}
Explicit bounds on the quantum partition functions are given in terms of classical partition functions, incorporating effective pair potentials, which account for Fermi- and Bose-statistics, respectively. The bounds may be used for the limit $\hbar \rightarrow 0$ and eventually for showing the interchangeability of the classical with the thermodynamic limit. A simple derivation of the thermodynamic limit for free particles with general dispersions is given.
\end{abstract}

\section{Introduction}

The correspondence principle for partition functions is quite an old problem. There exist heuristic discussions in text books and expansions in $\hbar$, but they are mathematically unsatisfactory. The problem may be divided into two points:

1) How is the trace over the Hilbert space related to the phase space integral?

2) How does the $1 / N$ ! for fermions and bosons originate?

The first question is settled in some detail by now [1-9], the second one is answered in this paper.

The main results appear in $2 d$ and $2 e$, equation (29), (30), (43). They concern the canonical ensemble and read, expressed in the free energy:

$$
F_{c l}\left(H_{c l}\right) \leqq F_{F}(H) \leqq F_{c l}\left(\hat{H}_{c l}+V_{F}(\hbar, \beta)\right) \text { for fermions }
$$

and

$$
F_{c l}\left(H_{c l}-V_{B}(\hbar, \beta)\right) \leqq F_{B}(H) \leqq F_{c l}\left(\hat{H}_{c l}\right) \text { for bosons }
$$

(cl stands for "classical", $F$ for fermions, $B$ for bosons, $\beta=1 / k T$ ). The Hamiltonians are supposed to be of the form

$$
H=\sum_{i=1}^{N} p_{i}^{2}+V\left(x_{1} \ldots x_{N}\right)
$$

and $H_{c l}$ is the same function of canonical coordinates instead of the operators $p_{i}, x_{i}$. Almost no condition restricts the set of allowed potentials $V$, one has only to make

* Work supported in part by Fonds zur Förderung der wissenschaftlichen Forschung in Österreich, Project No. 3569. 
sure, that the Hamiltonian and the partition functions are well defined, and that the Golden Thompson-inequality may be applied to $\exp (-\beta H)$. For the lower bound on the free energy the kinetic energy per particle may also be some other functions of its momentum. For the upper bound the configuration space has to be divided into cubes. The comparison Hamiltonian is

$$
\hat{H}_{c l}=\sum_{i=1}^{N}\left(p_{i}^{2}+c\right)+U\left(x_{1} \ldots x_{N}\right),
$$

with $U$ a stepfunction, which is constant on each cube and dominates $V$. The correction potentials $V_{F}$ and $V_{B}$ may heuristically be understood as effective pair interactions, which mimick the effect of quantum statistics. They go to zero in the limit $\hbar \rightarrow 0$ as well as $c$, and also $\hat{H}_{c l}$ converges to $H_{c l}$ in this limit, provided $V$ is sufficiently well behaved and one lets the diameter of the cubes approach zero more slowly than $\hbar$.

Provided that $H_{c l}-V_{B}$ is thermodynamically stable, which is the case, if $H_{c l}$ involves a sufficiently strong short range repulsion, all of the above bounds remain sensible in the thermodynamic limit. The interchangeability of the limit $N \rightarrow \infty$ and $\hbar \rightarrow 0$ posses therefore little problems, it is mainly a question of the continuity of $F_{c l}$ in $H_{c l}$. It is not treated here. The reader may consult [4] for those questions and also for the history of the subject.

To achieve some completeness, the proof of several well known results have been included: The use of coherent states for bounds on partition functions goes back to Lieb [5] and Berezin $[6,7]$. The original use of the Golden-Thompson inequality $[8,9]$ was just to give the bound of our section $2 a$, but the present proof is simpler than the original one.

The logical interrelations of the sections are the following: $1 a$ is needed for $1 b$ and $1 c, 1 d$ and $1 e$ are used in $2 e, 2 a$ gives the prerequisites for $2 c, 2 d$ and 3 . $2 d$, where the left hand sides of the above equations are derived, uses also the Appendix B.

The indices used throughout the paper are: $B, F, M B, c l$, to indicate Bose, Fermi, quantum mechanical Maxwell-Boltzmann or classical Maxwell-Boltzmann statistics, respectively. $N$ and $D$ indicate the presence of Neumann or Dirichlet boundary conditions.

\section{Microcanonical Ensemble}

The usual definition of the entropy as a function of the energy is

$$
S_{F, B}(E)=\log \operatorname{Tr}_{F, B} \theta(E-H)
$$

for fermions and bosons, respectively, and

$$
S_{M B}=\log \frac{1}{N !} \operatorname{Tr} \theta(E-H)
$$

for Maxwell-Boltzmann-Statistics. Since the $\theta$-function cannot be treated with our methods of part $a$ ) and $b$ ), we investigate rather the mean energy as a function of the 
entropy, defined for $S \varepsilon \log \mathbb{N}$

$$
\bar{E}(S)=\inf _{P_{S}} \operatorname{Tr} P_{S} H, \quad P_{S}=P_{S}^{*}=P_{S}^{2}, \quad \operatorname{Tr} P_{S}=e^{S} .
$$

We assume that the range of $P_{S}$ is in the space with the appropriate symmetry.

\section{a) A Lower Bound for Discernible Particles}

Let us assume that the Hamiltonian is operator-bounded below by $\mathrm{H}^{0}$, which allows for a diagonal representation with the coherent states (Appendix A):

$$
H \geqq H^{0}=\int \frac{d^{3 N} z}{(2 \pi \hbar)^{3 N}} H_{0}(z)|z\rangle\langle z| .
$$

We abbreviate the integral and normalization as $\int d \Omega(z)$. (2) becomes:

$$
\begin{aligned}
\bar{E}(S) & =\inf _{P_{S}} \operatorname{Tr} \int d \Omega(z) P_{S}|z\rangle H_{0}(z)\langle z|=\inf _{P_{S}} \int d \Omega(z)\left\langle z\left|P_{S}\right| z\right\rangle H_{0}(z) \\
& \geqq \inf _{f_{S}} \int d \Omega(z) f_{S}(z) H_{0}(z), \quad 0 \leqq f_{S}(z) \leqq 1, \quad \int d \Omega(z) f_{S}(z)=e^{S}
\end{aligned}
$$

(where we have used $\int d \Omega(z)\left\langle z\left|P_{S}\right| z\right\rangle=\operatorname{Tr} P_{S}$ ).

For that expression to become small, it is necessary that $f_{S}$ concentrates as much as possible at those $z$, where $H_{0}$ is small. Therefore one need only consider characteristic functions of sets $A_{S}$ :

$$
\bar{E}(S) \geqq E_{c l}(S)=\inf _{A_{S}} \int_{A_{S}} d \Omega(z) H_{0}(z), \quad \int_{A_{S}} d \Omega(z)=e^{S} .
$$

The inverse of the function $\bar{E}_{c l}(S)$ provides us with an upper bound on the number of states such that the sum of the energies does not exceed a prescribed value.

\section{b) A Lower Bound for Fermions}

Again we use (4), but with the additional restriction that $P_{S} \leqq P_{F}$, where $P_{F}$ is the projector onto the antisymmetrized wave-functions:

$$
P_{F}=\frac{1}{N !} \sum_{p \& \mathscr{G}(N)} \sigma(p) U_{p}, \quad\left(U_{p} \psi\right)\left(x_{1} \ldots x_{N}\right)=\psi\left(x_{p(1)} \ldots x_{p(N)}\right) .
$$

$(\mathscr{S}(N)$ is the group of permutations of $\{1,2, \ldots N\}, \sigma(p)$ is the sign of the permutation $p$.)

That implies:

$$
\left\langle z\left|P_{S}\right| z\right\rangle \leqq\left\langle\left|P_{F}\right| z\right\rangle=\frac{1}{N !} \sum_{p} \sigma(p) \prod_{i=1}^{N}\left\langle z_{i} \mid z_{p(i)}\right\rangle=\frac{1}{N !} \operatorname{det}\left\langle z_{i} \mid z_{j}\right\rangle \leqq \frac{1}{N !}
$$

(The $N$-particle coherent state $|z\rangle$ is a tensor-product of the 1-particle coherent states $\left|z_{i}\right\rangle$. For the last inequality see ref. [10]) 
And we arrive at

$$
\bar{E}_{F}(S) \geqq \inf _{A_{S}} \frac{1}{N !} \int_{A_{S}} d \Omega(z) H_{0}(z), \int_{A_{S}} d \Omega(z)=N ! e^{S}
$$

which with permutation symmetry of $H_{0}(z)$, is equivalent to using eq. (5) but with the restriction that

$$
\forall p \in \mathscr{S}(N), \quad p \neq i d:\left(x_{1} \ldots x_{N}\right) \in A_{S} \Rightarrow\left(x_{p(1)} \ldots x_{p(N)}\right) \notin A_{S} .
$$

c) Bounds for Non Interacting Discernible Particles in a Box

I a cubic box of length $\ell,(1)$ and (2) become, with $H=-\hbar^{2} \sum_{i=1}^{N} \Delta_{i}$

$$
\begin{aligned}
& S(E)=\log \sum_{\left\{n_{1} \ldots n_{3 N}\right\} \in Z_{+}^{3 N}} \theta\left(E-\hbar^{2} \frac{\pi^{2}}{\ell^{2}} \sum_{i=1}^{3 N} n_{i}^{2}\right), \\
& \bar{E}(S)=\inf _{A_{S} \in Z_{+}^{3 N}} \sum_{\left\{n \ldots n_{3 N}\right\} \in A_{S}}\left(\hbar^{2} \frac{\pi^{2}}{\ell^{2}} \sum_{i} n_{i}^{2}\right), \quad \sum_{A_{S}}=e^{S} .
\end{aligned}
$$

The sums may be regarded as integrals over step-functions: For Neumannboundary-conditions we associate each $\mathbf{n}=\left\{n_{1} \ldots n_{3 N}\right\},\left(n_{i} \varepsilon\{0,1,2, \ldots\}\right)$ with the cube $\left.\mathbf{x}: n_{i} \leqq x_{i} \leqq n_{i}+1\right\}$. For Dirichlet-boundary-conditions only those $\mathbf{n}$ are allowed, for which $n_{i} \neq 0$, and we associate $\mathbf{n}$ with the cube $\left\{\mathbf{x}: n_{i}-1 \leqq x_{i} \leqq n_{i}\right\}$. Formally:

$$
\left(\mathbf{n}_{N}(\mathbf{x})\right)_{i}=\left[x_{i}\right], \quad\left(\mathbf{n}_{D}(\mathbf{x})\right)_{i}=\left[x_{i}\right]+1
$$

which implies

$$
\left(\mathbf{n}_{N}\right)^{2} \leqq(\mathbf{x})^{2} \leqq\left(\mathbf{n}_{D}(\mathbf{x})\right)^{2}
$$

and

$$
\begin{aligned}
& S_{N}(E) \geqq S_{c l}(E)=\log V^{N} \int \frac{d^{3 N p}}{(2 \pi \hbar)^{3 N}} \theta\left(E-\sum p_{i}^{2}\right) \geqq S_{D}(E), \\
& \bar{E}_{N}(S) \leqq \bar{E}_{c l}(S) \leqq \bar{E}_{D}(S)
\end{aligned}
$$

where (with $V=\ell^{3}$ )

$$
\bar{E}_{c l}(S)=\inf _{A_{S} \in \mathbb{R}^{3 N}} V^{N} \int_{A_{S}} \frac{d^{3 N} p}{(2 \pi \hbar)^{3 N}}\left(p_{i}\right), \quad V^{N} \int_{A_{S}} \frac{d^{3 N} p}{(2 \pi \hbar)^{3 N}}=e^{S} .
$$

To obtain bounds in the other directions one may use Robinson's method of comparing Dirichlet- and Neumann-boundary-conditions [11]. $\bar{E}_{c l}$ evaluates to

$$
4 \pi \hbar^{2}\left(1+\frac{2}{3 N}\right)^{-1}\left(\Gamma\left(\frac{3 N}{2}+1\right)\right)^{2 / 3 N} V^{-2 / 3} e^{S(1+2 / 3 N)} .
$$




\section{d) Bounds for Non-Interacting Bosons}

Suppose the 1-particle states $\phi_{n}$ to be labelled by $n=1,2 \ldots$. Certain $N$-boson states may be labelled by $\left(n_{1}, n_{2} \ldots n_{N}\right)$ with $n_{i} \leqq n_{i+1}:{ }^{1}$ This stands for the symmetrized product state of $\phi_{n_{1}}$ which is an eigenstate of the Hamiltonian

$$
H_{N}=\sum_{i=1}^{N} h_{i}
$$

with eigenvalue $E=\sum \varepsilon_{n_{i}}$. (When $h \phi_{n}=\varepsilon_{n} \phi_{n}$.) We want to compare the situation with the case of discernible particles: There, the $N$-particle eigenstates are the tensor products of not necessarily different $\phi_{n_{l}}$ 's, and to a given collection of $n_{i}$ 's there exist $N ! / \prod_{n} k_{n}$ ! different eigenstates to the eigenvalue $E=\sum \varepsilon_{n_{l}} .\left(k_{n}\right.$ is the multiplicity of occupation of the level $n, \sum_{n} k_{n}=N$ and $1 \leqq N ! / \prod_{n} k_{n} ! \leqq N$ !.) Therefore

$$
\begin{aligned}
S_{B}(E)=\log \sum_{\substack{\left(n_{1}, \ldots, n_{N}\right) \\
n_{1} \leqq n_{2} \leqq \ldots}} \theta\left(E-\sum_{i} \varepsilon_{n_{i}}\right) & =\log \sum_{\substack{\left(n_{1}, \ldots, n_{N}\right) \\
\text { not ordered }}} \frac{\prod k_{n} !}{N !} \theta\left(E-\sum_{i} \varepsilon_{n_{i}}\right) \\
\geqq \log \frac{1}{N !} \sum_{\substack{\left(n_{1}, \ldots, n_{N}\right) \\
\text { not ordered }}} \theta\left(E-\sum_{i} \varepsilon_{n_{i}}\right) & =S_{M B}(E) .
\end{aligned}
$$

For particles in a box this may now be further developed into a classical bounds.

\section{e) Bounds for Non-Interacting Fermions}

As for the bosons, the $N$-fermion states may be labelled by $\left(n_{1} \ldots n_{N}\right)$, but with $n_{i}$ strictly smaller than $n_{i+1}$. This restriction forces us to find another correspondence between the fermion states and groups of indiscernible-particle-states: The group of $N ! / \prod k_{n}$ ! product-states with the occupied levels $\left(n_{1}, n_{2} \ldots n_{N}\right)$ (where the $n_{i}$ are ordered) is now compared to the fermion state $\left(n_{1}, n_{2}+1, n_{3}+2 \ldots n_{N}+N-1\right)$, which has the energy

$$
\begin{aligned}
& \sum_{i} \varepsilon_{n_{i}}+\left(\varepsilon_{n_{2}+1}-\varepsilon_{n_{2}}\right)+\left(\varepsilon_{n_{3}+2}-\varepsilon_{n_{3}}\right)+\ldots+\left(\varepsilon_{n_{N}+N-1}-\varepsilon_{n_{N}}\right) \\
& \quad \leqq \sum_{i} \varepsilon_{n_{l}}+\frac{N(N-1)}{2} \sup _{n}\left(\varepsilon_{n+1}-\varepsilon_{n}\right) .
\end{aligned}
$$

For particles in a 3-dimensional cubic box of length $\ell$ it follows from number-theory that indeed

$$
\sup \left(\varepsilon_{n+1}-\varepsilon_{n}\right) \leqq \Delta=5 \frac{\hbar^{2} \pi^{2}}{\ell^{2}}
$$

is finite (Appendix C). For Neumann-boundary-conditions the 5 may be replaced by

1 The $n_{i}$ 's are not the usual occupation numbers! 
3. (We conjecture that in 3 space dimensions that sup is also finite, if regions of a more general shape are chosen.) The extra term in the energy is the same as would be present as the effect of a mutual interaction with strength $\Delta$ and range at least the diameter of the volume.

$$
S_{F}(E) \geqq \log \frac{1}{N} \sum_{\substack{\left(n_{1} \ldots n_{N}\right) \\ \text { not ordered }}} \theta\left(E-\sum_{i} \varepsilon_{n_{l}}-\frac{N(N-1)}{2} \Delta\right)=S_{M B}\left(E-\frac{N(N-1)}{2} \Delta\right)
$$

Without correction, the bound is in the other direction, since each fermion state corresponds to $N$ ! states of indiscernible particles with the same energy (but not all states of the indiscernible particles are reached in this direct way):

$$
S_{M B}(E) \geqq S_{F}(E) .
$$

It has to be remarked that the above method works only in at least three space dimensions. A more general upper bound for the energy may be found by another correspondence of groups of eigenvalues of discernible particles to the eigenvalues of the fermion system: We "forbid" the discernible particles to occupy the $N-1$ lowest levels and consider for them the partition function with $H=\sum h_{i}$ replaced by $\sum h_{i} \theta\left(h_{i}-\varepsilon_{N}\right)$. The group of eigenstates for which the levels $\left(n_{1}, \ldots, n_{N}\right), N \leqq n_{i}$ $\leqq n_{i+1}$, are occupied has higher energy than the fermion state $\left(n_{1}-(N\right.$ $\left.-1), \ldots, n_{N-1}-1, n_{N}\right)$ and therefore

$$
S_{F}(E) \geqq \log \frac{1}{N !} \sum_{\substack{\left(n_{1} \ldots n_{N}\right) \\ \text { not ordered, } n_{i} \geqq N}} \theta\left(E-\sum_{i} \varepsilon_{n_{l}}\right),
$$

and

$$
\begin{aligned}
& \bar{E}_{F}(S) \leqq \inf _{A_{S}} \frac{1}{N !} \sum_{\left(n_{1} \ldots n_{N}\right) \in A_{S}}\left(\sum_{i} \varepsilon_{n_{l}}\right), \quad\left(n_{1} \ldots n_{N}\right) \in A_{S} \Rightarrow n_{i} \geqq N, \\
& \sum_{\left(n_{1} \ldots n_{N}\right) \in A_{S}}=N ! e^{S}
\end{aligned}
$$

\section{The Canonical Ensemble}

a) Discernible Particles: Upper Bound to the Partition Function

The Hamiltonian is supposed to be of the form $H=K(p)+V(x), e^{-\beta K(p)}$ defining an integral kernel in $x$-space:

$$
\left(e^{-\beta K} \psi\right)(x)=\int K_{K}(x-y) \psi(y) d^{3 N} y .
$$

We will make use of the fact that $K_{K}(0)$ is just the classical phase-space integral:

$$
K_{K}(0)=\left(\frac{1}{2 \pi \hbar}\right)^{3 N} \int d^{3 N} p e^{-\beta K(p)}
$$

An upper bound to the partition function is found by use of the Golden-Thompson 
inequality:

$$
\begin{aligned}
N ! Q_{M B}(\beta): & =\operatorname{Tr} e^{-\beta(K+V)} \leqq \operatorname{Tr} e^{-\beta K} e^{-\beta V}=\int d^{3 N} x K_{K}(0) e^{-\beta V(x)} \\
& =\int \frac{d^{3 N} x d^{3 N} p}{(2 \pi \hbar)^{3 N}} e^{-\beta H_{c l}(p, x)}
\end{aligned}
$$

The classical Hamiltonian is defined by replacing the $p$ - and $x$-operators by coordinates.

The following bound goes back to Lieb [5]. It has the disadvantage not to be immediately applicable to all Hamiltonians (this topic is discussed in the Appendix A). Furthermore, the bound is weaker than (19). On the other hand it remains valid, when the exponential is replaced by any convex and decreasing function, and for other types of coherent states $[5,4]$.

We suppose, that the Hamiltonian has an operator-bound below, which is representable by the coherent states as

$$
H^{0}=\int d \Omega(z)|z\rangle H_{0}(z)\langle z| \leqq H .
$$

Denote the complete set of eigenvectors of $H$ as $\left\{\psi_{n}\right\}$. One has

$$
\operatorname{Tr} e^{-\beta H}=\sum_{n} \exp \left(-\beta\left\langle\psi_{n}|H| \psi_{n}\right\rangle\right),
$$

and since the exponential is a monotone function:

$$
\begin{aligned}
& \exp \left(-\beta\left\langle\psi_{n}|H| \psi_{n}\right\rangle\right) \geqq \exp \left(-\beta\left\langle\psi_{n}\left|H^{0}\right| \psi_{n}\right\rangle\right) \\
& \quad=\exp \left(-\int d \Omega(z)\left|\left\langle\psi_{n} \mid z\right\rangle\right|^{2} \beta H_{0}(z)\right) .
\end{aligned}
$$

Since $\int d \Omega(z)\left|\left\langle\psi_{n} \mid z\right\rangle\right|^{2}=\left\|\psi_{n}\right\|^{2}=1$, we may use the convexity of the exponential:

$$
N ! Q_{M B}(\beta) \leqq \int d \Omega(z) \sum_{n}\left|\left\langle\psi_{n} \mid z\right\rangle\right|^{2} e^{-\beta H_{0}(z)}=\int d \Omega(z) e^{-\beta H_{0}(z)}
$$

\section{b) Discernible Particles: Lower Bound to the Partition Function}

With the completeness of the coherent states and again by the convexity of the exponential one has [5]:

$$
N ! Q_{M B}(\beta)=\int d \Omega(z) \operatorname{Tr}|z\rangle\langle z| e^{-\beta H} \geqq \int d \Omega(z) e^{-\beta\langle z|H| z\rangle} .
$$

\section{c) Bounds with Quantum Statistics, Involving the Coherent States}

We use the form of the completeness relation which is valid in the $N$-particle-space for bosons and fermions (Appendix A), due to Thirring [15]:

$$
\left.\mathbb{1}_{B, F}=\frac{1}{N !} \int d \Omega(z) \prod_{i=1}^{N} a^{*}\left(z_{i}\right) \mid \Omega\right)\left(\Omega \mid \prod_{i=1}^{N} a\left(z_{i}\right)\right.
$$

and denote $\left.\left.\prod a^{*}\left(z_{i}\right) \mid \Omega\right)=\mid z_{1} \ldots z_{N}\right)=\mid z$ ). These states are not normalized: For fermions it is known that by the Hadamard-theorem [10]

$$
(z \mid z)_{F}=N !\left\langle z\left|P_{F}\right| z\right\rangle=\operatorname{det}\left\langle z_{i} \mid z_{j}\right\rangle \leqq 1 .
$$


For bosons by the Marcus-theorem [12]

$$
(z \mid z)_{B}=N !\left\langle z\left|P_{B}\right| z\right\rangle=\operatorname{per}\left\langle z_{i} \mid z_{j}\right\rangle \leqq 1 .
$$

This has to be remembered when one uses the convexity of the exponential:

$$
Q_{F, B}(\beta) \geqq \frac{1}{N !} \int d \Omega(z)(z \mid z) e^{-\beta(z|H| z) /(z \mid z)} .
$$

It is not easy to write down the scalar products and expectation values as functions of $z$. But it may be seen that, for $N$ fixed, in the classical limit, $(z \mid z)$ converges to 1 and $(z \mid \mathrm{Hz})$ to the classical Hamiltonian almost everywhere. Everywhere for bosons, for fermions for those $z$, where all $z_{i}$ are different.

If one uses coherent states with compact support, that is to say, $\left|z_{i}\right\rangle$ is given for $z_{i}=0$ by the wave function $\phi\left(x_{i}\right)$ and the support of $\phi(x)$ is $D$, a set with finite diameter $\ell$, then one has in the case, that all $\left|r_{i}-r_{j}\right| \geqq \ell(r$ is the space coordinate of $z$ ) $(z \mid z)=1$, and by the locality and symmetry of $H$ also $(z|H| z)=\langle z|H| z\rangle$. One gets a simple bound by restricting the integrations to this region:

$$
Q_{F, B}(\beta) \geqq \frac{1}{N !} \int_{\left|r_{\iota}-r_{\jmath}\right| \geqq l} d \Omega(z) \exp (-\beta\langle z|H| z\rangle) .
$$

This restriction simulates a hard core potential, and the r.h.s. of (25) equals the classical partition function with the Hamiltonian

$$
\hat{H}(p, x)=\left\{\begin{array}{cc}
\sum_{i} \int q^{2}\left|\tilde{\phi}\left(q-p_{i}\right)\right|^{2} d^{3} q+\int V\left(y_{1} \ldots y_{N}\right) \prod_{i}\left|\phi\left(y_{i}-x_{i}\right)\right|^{2} d^{3 N} y_{i} \\
\quad \text { if all }\left|x_{i}-x_{j}\right|>\ell \\
\infty \quad & \text { if some }\left|x_{i}-x_{j}\right| \leqq \ell
\end{array}\right.
$$

$\tilde{\phi}$ is the Fourier transformed $\phi$.

If $V\left(x_{1} \ldots x_{N}\right)=\sum_{i<j} V\left(x_{i}-x_{j}\right)$, then

$$
\begin{aligned}
& \hat{H}(p, x)=\sum_{i}\left(p_{i}^{2}+2 p_{i}\langle\phi|p| \phi\rangle+\left\langle\phi\left|p^{2}\right| \phi\right\rangle+\sum_{i<j} \hat{V}\left(x_{i}-x_{j}\right),\right. \\
& \hat{V}(x)= \begin{cases}\int_{\infty} V(x-y)|\phi(z-y)|^{2}|\phi(-z)|^{2} d^{3} z d^{3} y, & |x|\rangle \ell \\
\infty & |x| \leqq \ell .\end{cases}
\end{aligned}
$$

For the upper bound, we make the observation, that the Hamiltonians are often in a form, which allows to regard them as operating on the whole- $N$-particle space without restriction from the statistics. We assume therefore (20) and write the partition function as a trace over the whole space, but including the appropriate projection operator, given by eq. (6) for fermions and similarly for bosons (without the $\sigma(p))$. One gets analogously to eq. (21):

$$
Q_{B, F}(\beta):=\operatorname{Tr} P_{B, F} e^{-\beta H} \leqq \int d \Omega(z)\left\langle z\left|P_{B, F}\right| z\right\rangle e^{-\beta H_{0}(z)} .
$$


We may use (23a):

$$
Q_{F}(\beta) \leqq \frac{1}{N !} \int d \Omega(z) e^{-\beta H_{0}(z)} .
$$

For bosons we use the corollary from Appendix B:

$$
Q_{B}(\beta) \leqq \frac{1}{N !} \int d \Omega(z) \exp \left(-\beta H_{0}(z)+\sum_{i<j}\left|\left\langle z_{i} \mid z_{j}\right\rangle\right|\right) .
$$

d) Bounds with Quantum Statistics, Involving the Golden-Thompson-Inequality The kinetic energy is supposed to be of the form $K(p)=\sum_{i} k\left(p_{i}\right), e^{-\beta k}$ giving rise to an integral kernel $K_{K}(x-y)$, which is positive definite, since $e^{-\beta K}$ is a positive operator. Therefore, for any $N$ and $\left(x_{1} \ldots x_{N}\right)$, the matrix $\left(m_{i j}\right)=\left(K_{k}\left(x_{i}-x_{j}\right)\right)$ is positive definite:

$$
\begin{aligned}
Q_{F}(\beta) & \leqq \operatorname{Tr} P_{F} e^{-\beta K} e^{-\beta V}=\frac{1}{N !} \int d^{3 N} x \operatorname{det}\left(m_{i j}\right) e^{-\beta V(x)} \\
& \leqq \frac{1}{N !} \int d^{3 N} x\left(K_{k}(0)\right)^{N} e^{-\beta V(x)}=\frac{1}{N !} \int \frac{d^{3 N} x d^{3 N} p}{(2 \pi \hbar)^{3 N}} e^{-\beta H_{c l}(p, x)} .
\end{aligned}
$$

For the first inequality in (29) as well as for the corresponding step in (30), it is of course essential, that not only $K+V$, but also $K$ and $V$ separately commute with the appropriate projector $P_{F}$ or $P_{B}$.

For bosons, we use the corollary of Appendix B for the matrix $a_{i j}(x)=$ $K_{k}\left(x_{i}-x_{j}\right) / K_{k}(0)$ :

$$
\begin{aligned}
Q_{B}(\beta) & \leqq \frac{1}{N !} \int d^{3 N} x\left(K_{k}(0)\right)^{N} \operatorname{per}\left(a_{i j}(x)\right) e^{-\beta V(x)} \\
& \leqq \frac{1}{N !} \int \frac{d^{3 N} x d^{3 N p}}{(2 \pi \hbar)^{3 N}} \exp \left(-\beta\left(H_{c l}(p, x)+\sum_{i<j} V_{B}\left(x_{i}-x_{j}\right)\right)\right)
\end{aligned}
$$

The effect of the bose-statistics is here estimated by the effect of an attracting interaction-potential $V_{B}(x)=\frac{1}{\beta} K_{k}(x) / K_{k}(0)$. For the non-relativistic kinetic energy, $k=p^{2}$, it is

$$
V_{B}(x)=\frac{1}{\beta} \exp \left(-\frac{1}{4 \beta \hbar^{2}}|x|^{2}\right) .
$$

e) Bounds with Quantum Statistics, Involving Jensen's Inequality

We assume in this section, that $H=K+V, K=\sum p_{i}^{2}, V=V\left(x_{1} \ldots x_{N}\right)$. For any orthonormal set of wave functions $\left\{\psi_{n}\right\}$ one has

$$
\operatorname{Tr} e^{-\beta H} \geqq \sum_{n} \exp \left(-\beta\left\langle\psi_{n}|H| \psi_{n}\right\rangle\right) .
$$

We divide the physical space into cubes with sidelength $\ell$, denote their centres by $\xi$ 
and choose as $\left(\psi_{n}\right\}$ the eigenfunctions of the kinetic energy with Dirichlet boundary conditions on the faces of the cubes. Such a $\psi$ may be characterized by the numbers of particles in each cell, $\rho(\xi)$ (a non-negative integer) and in addition by the occupied levels in each cell $\left(n_{1} \ldots n_{\rho(\xi)}\right)_{\xi}$ :

$$
\begin{aligned}
\operatorname{Tr} e^{-\beta H} \geqq & \left.\sum_{\{\rho(\xi)\}} \sum_{\left\{\left(n_{1}\right)_{\xi}\right\}} \exp \left(-\beta K\left[p,\left\{n_{i}\right)_{\xi}\right\}\right]-\beta V\left[\rho,\left\{\left(n_{i}\right)_{\xi}\right\}\right]\right), \\
& \sum_{\xi} \rho(\xi)=N \\
& K\left[\rho,\left\{\left(n_{i}\right)_{\xi}\right\}\right]=\sum_{\xi} \sum_{i=1}^{\rho(\xi)} \varepsilon_{n_{i}(\xi)}, \\
& V\left[\rho,\left\{\left(n_{i}\right)_{\xi}\right\}\right]=\left\langle\psi_{\rho,\left(n_{i}\right)}|V| \psi_{\rho,\left(n_{i}\right)}\right\rangle
\end{aligned}
$$

The $\varepsilon_{n}$ 's constitute the spectrum of $-\hbar^{2} \Delta_{D}$ on $L^{2}(C), C$ a cube with sidelength $\ell$. Firstly, we aim at removing the dependence of $V\left[\rho,\left\{\left(n_{i}\right)_{\xi}\right\}\right]$ upon the levels and at giving an upper bound to it in the form $U[\rho]$. We define the comparison potential $U$ as the step function

$$
U\left(x_{1}, \ldots, x_{N}\right)=U\left(\xi\left(x_{1}\right), \ldots, \xi\left(x_{N}\right)\right)
$$

where $\xi(x)$ denotes the center of that cube $C(\xi)$, in which $x$ is located (the boundaries are inessential), and

$$
U\left(\xi_{1} \ldots \xi_{N}\right)=\sup _{x_{1} \in C\left(\xi_{1}\right) \ldots x_{N} \in C\left(\xi_{N}\right)} V\left(x_{1} \ldots x_{N}\right) .
$$

If this sup happens to be infinite, it may nevertheless be used consistently in the sequel with the definition $e^{-\infty}=0$. $U$ dominates $V$ pointwise, and obviously

$$
V\left[\rho,\left\{\left(n_{i}\right)_{\xi}\right\}\right] \leqq U\left[\rho,\left\{\left(n_{i}\right)_{\xi}\right]=U[\rho] .\right.
$$

Since the contributions to $K\left[\rho,\left\{\left(n_{i}\right)_{\xi}\right\}\right]$ from different cells are additive, and since the occupation numbers $\left(n_{i}\right)$ of different cells may vary independently of each other, one may rewrite the r.h.s. of (32) with $V$ replaced by $U$ as

$$
\sum_{\{\rho(\xi)\}} \exp (-\beta U[\rho]) \prod_{\xi} \operatorname{Tr}_{\xi, \rho(\xi)} \exp \left(-\beta \sum_{i=1}^{\rho(\xi)} p_{D, i}^{2}\right) .
$$

$\operatorname{Tr}_{\xi, \rho}$ means the trace in the space of (anti) symmetrized wave functions for $\rho$ particles in the cube $C(\xi)$.

$U[\rho]$ is the potential energy $U\left(\xi_{1} \ldots \xi_{N}\right)$ of any lattice configuration $\left(\xi_{1} \ldots \xi_{N}\right)$ which has the lattice occupation numbers $\rho(\xi)$. Adopting the convention $0 !=1$, there are $N !\left(\prod_{\xi} \rho(\xi) !\right)^{-}$such lattice configurations. The sum over $\{\rho(\xi)\}$ may therefore be transformed into a sum over configurations $\left(\xi_{1} \ldots \xi_{N}\right)$ :

$$
\begin{aligned}
\operatorname{Tr} e^{-\beta H} & \geqq \frac{1}{N !} \sum_{\left(\xi_{1} \ldots \xi_{N}\right)} \exp \left(-\beta U\left(\xi_{1} \ldots \xi_{N}\right) \prod_{\xi} \rho(\xi) ! \operatorname{Tr}_{\xi, \rho} \exp \left(-\beta \sum_{i=1}^{\rho(\xi)} p_{D, i}^{2}\right)\right. \\
& =\frac{1}{N !} \sum_{\left(\xi_{1} \ldots \xi_{N}\right)} \exp \left(-\beta U\left(\xi_{1} \ldots \xi_{N}\right)-\beta \sum_{i} F\left(\beta, \rho\left(\xi_{i}\right)\right) .\right.
\end{aligned}
$$


In the second step we have introduced a modified free energy of $\rho$ particles in a cube $C(\xi)$ :

$$
F(\beta, \rho)=-\frac{1}{\beta} \log \left(\rho ! \operatorname{Tr}_{\xi, \rho} \exp \left(-\beta \sum_{i=1}^{\rho} p_{D, i}^{2}\right)\right)
$$

which is bounded from above by the free energy of discernible particles, using the methods of $1 d$ and $1 e$ ):

$$
\begin{aligned}
& F(\beta, \rho) \leqq-\frac{1}{\beta} \log \operatorname{Tr}_{\xi, \rho} e^{-\beta K_{D}}+\frac{\rho(\rho-1)}{2} \Delta, \\
& \Delta= \begin{cases}0 & \text { for bosons } \\
5 \hbar^{2} \pi^{2} / \ell^{2} & \text { for fermions. }\end{cases}
\end{aligned}
$$

The trace is here over the Hilbert space for discernible particles. The extra term for fermions may be written as a pair interaction

$$
\begin{aligned}
& V_{F}(x, y)=V_{F}(\xi(x), \xi(y)), \\
& V_{F}(\xi, \eta)= \begin{cases}0 & \xi \neq \eta \\
\Delta & \xi=\eta .\end{cases}
\end{aligned}
$$

Writing $F_{D}(\beta, \rho)=-\frac{1}{\beta} \log \operatorname{Tr} e^{-\beta K_{D}}$, we have:

$$
\operatorname{Tr} e^{-\beta H} \geqq \frac{1}{N !} \sum_{\xi_{1} \ldots \xi_{N}} \exp \left(-\beta F_{D}\left(\beta, \rho\left(\xi_{i}\right)\right)-\beta \sum_{i<j} V_{F}\left(\xi_{i}, \xi_{j}\right)-\beta U\left(\xi_{1} \ldots \xi_{N}\right)\right) .
$$

But for discernible non-interacting particles the free energy is a sum over the free energies of the individual particles:

$$
\begin{aligned}
& F_{D}(\beta, \rho)=\rho f(\beta), \quad f(\beta)=-\frac{1}{\beta} \log \operatorname{Tr}_{1} e^{-\beta p_{D}^{2}} \\
& \operatorname{Tr} e^{-\beta H} \geqq \frac{1}{N !} \sum_{\xi_{1} \ldots \xi_{N}} \exp \left(-\beta\left(\sum_{i} f(\beta)+\sum_{i<j} V_{F}\left(\xi_{i}, \xi_{j}\right)+U\left(\xi_{1} \ldots \xi_{N}\right)\right)\right) .
\end{aligned}
$$

For $f(\beta)$ one finds upper bounds:

$$
\begin{aligned}
& f(\beta)=-\frac{1}{\beta} \log \left(\ell^{3} \int \frac{d^{3} p}{(2 \pi \hbar)^{3}} e^{-\beta p^{2}}\right)+c(\beta, \hbar, \ell), \\
& c(\beta, \hbar, \ell) \leqq \begin{cases}\pi \hbar^{2} / \ell^{2} & \text { if } \beta \hbar^{2} / \ell^{2} \geqq 4 / \pi \\
16 \pi / \beta & \text { if } \beta \hbar^{2} / \ell^{2} \leqq 4 / \pi \\
-\frac{1}{\beta} \log \left(1-3 \frac{\pi \hbar}{\ell} \sqrt{\pi \beta}\right) & \text { if } \beta \hbar^{2} / \ell^{2}<1 / 9 \pi^{3} .\end{cases}
\end{aligned}
$$

Now, one may reinterpret the sum over the $\xi_{i}$ together with $\ell^{3 N}$ as integral over the 
step functions:

$$
\operatorname{Tr} e^{-\beta H} \geqq \frac{1}{N !} \int d \Omega(p, x) \exp \left(-\beta\left(\sum_{i} p_{i}^{2}+\sum_{i \leq j} V_{F}\left(x_{1}, x_{j}\right)+U\left(x_{1} \ldots x_{N}\right)+c N\right)\right) .
$$

If $U$ is a stable potential, that bound has a thermodynamic limit, which commutes with the classical limit, if the classcial free-energy-density is continuous in the limit $U \rightarrow V$. This is the case, if Simon's microstability contition holds ([4]).

\section{The Grand Canonical Ensemble}

For mutually interacting particles one may use the results of part 2) to bound each single term of

$$
\Xi(z)=\sum_{N} z^{N} Q(\beta, N)
$$

For noninteracting particles, only the upper bounds for fermions and the lower bounds for bosons may be summed up explicitly, and they give the bound by classical MB-Statistics. One can do better:

The partition function may be expressed as a function of the one-particleHamiltonian:

$$
\Xi(z)_{B, F}=\operatorname{Tr} f_{B, F}(h), \quad f_{B, F}(h)= \pm \log \left(1 \pm z e^{-\beta h}\right) .
$$

These are convex functions and the coherent states, $|w\rangle=|r, s\rangle$, may be used successfully:

$$
\left.\int \frac{d^{3} r d^{3} s}{(2 \pi \hbar)^{3}} f_{B, F} \mid\langle w|h| w\rangle\right) \leqq \operatorname{Tr} f_{B, F}(h) \leqq \int \frac{d^{3} r d^{3} s}{(2 \pi \hbar)^{3}} f_{B, F}\left(h_{0}(w)\right) .
$$

For bosons, one has a slightly better upper bound: expand $f_{B}(h)=\sum_{n} \frac{z^{n}}{n} e^{-\beta n h}$, bound each term with the help of the Golden-Thompson-inequality and sum up the series:

$$
\operatorname{Tr} f_{B}(h) \leqq \int \frac{d^{3} p d^{3} x}{(2 \pi \hbar)^{3}} f_{B}\left(h_{c l}(p, x)\right)
$$

The interchange of the expansion with both the trace and the integral is allowed, in view of the Lebesgue dominated convergence theorem.

Eq. (45) is not only stronger than the r.h.s. of eq. (44), it is also applicable to a wider class of Hamiltonians. It may be used for photons, where $h(p, x)=|p|+V(x)$, for example, and for $V(x)$ unbounded from below.

The differences between upper and lower bounds vanish in the thermodynamic limit for free particles, provided one lets the coherent states vary as $\psi_{\ell}(x)$ $=\psi\left(\ell^{-1 / 2} x\right)$, where $\ell$ is a characteristic length of the system. This is therefore an elegant way to derive the thermodynamic functions for free particles: Whenever the kinetic energy is $k(p)$, where $k$ is not too wild $\left(a \mathscr{C}^{1}\right.$-function with polynomial growth, 
for example):

$$
\lim _{V \rightarrow \infty} \frac{1}{V} \Xi(z)_{B, F}= \pm \int \frac{d^{3} p}{(2 \pi \hbar)^{3}} \log \left(1 \pm e^{-\beta k(p)}\right) .
$$

\section{Appendix A. On the Coherent States}

The basic definitions are as follows [13]: Let $\phi(x)$ be any normalized $\mathscr{L}^{2}\left(\mathbb{R}^{n}\right)$ function, denote it by $|0\rangle$ and define

$$
|z\rangle:=W(z)|0\rangle
$$

where $W(z)$ is the Weyl-operator:

$$
W(z)=e^{-i r s / 2} e^{i r p} e^{i s x}, \quad z=(r, s),
$$

$r$ and $s$ vectors of $\mathbb{R}^{n}$.

Then the identity on $\mathscr{L}^{2}\left(\mathbb{R}^{n}\right)$ is $\mathbb{1}=\int d \Omega(z)|z\rangle\langle z|$.

Consider an operator $H$ on $\mathscr{L}^{2}\left(\mathbb{R}^{n}\right)$ and suppose, for the moment, that it is given as

$$
H=\int d \Omega(z) H_{0}(z)|z\rangle\langle z| .
$$

Then, if all $w$ are in the form domain of $H$, define

$$
H(w):=\langle w|H| w\rangle=\int d \Omega(z) \rho(w-z) H_{0}(z)
$$

where $\rho(z)=|\langle 0 \mid z\rangle|^{2}$, and the Fourier transform of A.2 is

$$
\tilde{H}(u)=\tilde{\rho}(u) \tilde{H}_{0}(u) \text {. }
$$

This formula makes sense, if $H_{0}(z)$ is only polynomially increasing. $\tilde{H}_{0}$ is then a tempered distribution. When does the inversion of A.2,

$$
\tilde{H}_{0}(u)=\tilde{H}(u) / \tilde{\rho}(u)
$$

make sense? If $\phi(x)$ is a Gaussian, then also $\tilde{\rho}$ is a Gaussian, and $\tilde{H} / \tilde{\rho}$ will be no longer only polynomially increasing, for general $H$. But it is certainly so, if $\tilde{H}$ is a distribution with compact support. This is the case, by the Paley-Wiener theorem [14], if $H(w)$ has an extension to an entire analytic function of exponential type. $H_{0}$ is then a function of the same type. This is a sufficient and clearly not a necessary condition, but it covers the cases, where $H$ is a polynomial in $p, x$ and several $\exp \left(i k_{i} x\right)$, for instance.

Now consider $H=p^{2}+V(x)$. A representation of $p^{2}$ in the form

$$
p^{2}=\int d \Omega(z)|z\rangle K(s)\langle z|
$$

exists, if $\phi$ is in the operator domain of $p$ and is given by

$$
K(s)=s^{2}+2\langle\phi \mid p \phi\rangle s+2\langle\phi \mid p \phi\rangle^{2}-\langle p \phi \mid p \phi\rangle .
$$

Similarly, the operator identity

$$
V(x)=\int d \Omega(z)|z\rangle V_{0}(r)<z \mid
$$


holds for

$$
V(x)=\int V_{0}(r)|\phi(x-r)|^{2} d^{n} r .
$$

It is either difficult or impossible to find the representation in this form for usual $V$, but for $V$ bounded from below one can find bounds: Take an absolutely continuous $\phi$ with compact support in the domain $D$, and define

$$
W_{0}(x)=\inf _{y \varepsilon D} V(x-y),
$$

then

$$
\int d(z)\left|z>W_{0}(r)<z\right| \leqq V .
$$

The following version of coherent states for bosons and fermions is due to $W$. Thirring [15]: Set

$$
\begin{aligned}
& a^{*}\left(z_{i}\right)=\int a^{*}(x)\left(W\left(z_{i}\right) \phi\right)(x) d^{n} x, \\
& \left.|z\rangle=\prod_{i=1}^{N} a^{*}\left(z_{i}\right) \mid \Omega\right),
\end{aligned}
$$

where $\mid \Omega$ ) is the no particle state. Then

$$
\mid z)_{B, F}=\sqrt{N !} P_{B, F}\left|z_{1} \ldots z_{N}\right\rangle
$$

where $\left|z_{1} \ldots z_{N}\right\rangle$ is the unsymmetrized product of the $\left|z_{i}\right\rangle$. We have then the following representation of the identity in $N$-boson or fermion space as the projector $P_{B}$ or $P_{F}$ :

$$
1_{B, F}=P_{P, F} \int d \Omega(z)|z\rangle\left\langle z\left|P_{B, F}=\frac{1}{N !} \int d \Omega(z)\right| z\right)(z \mid .
$$

\section{Appendix B. On Permanents}

The permanent is defined as

$\operatorname{per} A=\sum_{p \in \mathscr{S}(N)} \prod_{i=1}^{N} a_{i, p(i)}$.

It admits an expansion quite similar to the one for determinants: Denote by ${ }^{0} A_{k, l}$ the $(N-1) \times(N-1)$ submatrix of A without the $k^{\prime}$ th column and $l$ th row: $\left({ }^{0} A_{k l}\right)_{i j}=a_{i j}$, $i \neq k, j \neq l$. The expansion formula is

$$
\text { per } A=\sum_{k=1}^{N} a_{k l} \operatorname{per}^{0} A_{k l}
$$

for any $l$.

In considering upper bounds one may use as a first step that

$$
\text { per } C \geqq \operatorname{per} A, \quad \text { if } \quad c_{i j}=\left|a_{i j}\right|,
$$

and treat in the sequel matrices with positive elements. 
Theorem. Let $A$ be an $N \times N$ matrix with $0 \leqq a_{i j}=a_{j l} \leqq a_{i i}=1$ for all $i$, $j$. One has the inequality

$$
\operatorname{per} A \leqq \prod_{1 \leqq i<j \leqq N}\left(1+a_{i j}\right)
$$

Proof. Replace the l'th row of $A$ by the row $(1,1, \ldots, 1)$ and denote this matrix as $P_{l}(A)$. Because all $a_{i j}$ are positive, per $P_{l}(A) \geqq \operatorname{per} A$ holds. Now one proves

$$
\operatorname{per} P_{l}(A) \leqq \prod_{i, j, i<j}\left(1+a_{i j}\right)
$$

by induction on the rank $N$ :

a) For $N=1$, A equals $P_{l}(A)=1$, and per $1=1$.

b) Suppose (B.4) to hold for $(N-1) \times(N-1)$ matrices and use the expansion (B.2) for the $N \times N$ matrix $P_{l}(A)$ :

$$
\text { per } P_{l}(A)=\sum_{k=1}^{N} a_{k l}{ }^{0}\left(P_{l}(A)\right)_{k l} \text {. }
$$

Now, ${ }^{0}\left(P_{l}(A)\right)_{l l}={ }^{0} A_{l l}$ and, for $k \neq l$ :

${ }^{0}\left(P_{l}(A)\right)_{k l}=P_{l}\left({ }^{0} A_{k l}\right)$.

${ }^{0} A_{k l}$ is not a symmetric matrix, but ${ }^{0} A_{l l}$ is, and $P_{l}\left({ }^{0} A_{k l}\right)$ differs from $P_{k}\left({ }^{0} A_{l l}\right)$ only by the interchange of two rows, an operation, which has no effect upon the permanent. (This is immediate from the definition B.1) For $P_{k}\left({ }^{0} A_{l l}\right)$ B.4 applies:

$$
\operatorname{per}^{0}\left(P_{l}(A)\right)_{k l}=\operatorname{per} P_{k}\left({ }^{0} A_{l l}\right) \leqq \prod_{i, j, i<j, i \neq l, j \neq l}\left(1+a_{i j}\right)
$$

Since the r.h.s. does not depend upon $k$, and since

$$
\sum_{k} a_{k l}=1+\sum_{k, k \neq l} a_{k l} \leqq \prod_{k, k \neq l}\left(1+a_{k l}\right)
$$

this inequality together with (B.6) used in (B.5) gives (B.4). q.e.d.

Corollary. If $A$ is a symmetric matrix with $\left|a_{i j}\right| \leqq 1$, then by (B.3):

$$
\operatorname{per} A \leqq \prod_{i \leqq j}\left(1+\left|a_{i j}\right|\right)
$$

and, since $1+x \leqq e^{x}$ :

$$
\text { per } A \leqq \exp \left(\sum_{i<j}\left|a_{i j}\right|\right) \text {. }
$$

The following simpler proof of the weakened, but generally valid inequality (with restricted validity first proven by Thirring)

$$
\text { per } A \leqq \exp \left(\sum_{i, j}\left|a_{i j}\right|\right)
$$


is due to $A$. Pflug (private communication):

$$
\operatorname{per} A \leqq \sum_{p \in \mathscr{S}(N)} \prod_{i=1}^{N}\left|a_{i, p(i)}\right| \leqq \prod_{i, j}\left(1+\left|a_{i j}\right|\right) \leqq \prod_{i, j} \exp \left|a_{i j}\right| \text {. }
$$

The second inequality arises as follows: The product of all $\left(1+\left|a_{i j}\right|\right)$ is equal to a sum over all subsets $M$ of the set of pairs $\{1 \ldots \mathrm{N}\} \times\{1 \ldots \mathrm{N}\}$ :

$$
\prod_{i, j}\left(1+\left|a_{i j}\right|\right)=\sum_{M \in\{1 \ldots N\} \times\{1 \ldots N\}} \prod_{(i, j) \in M}\left|a_{i j}\right| .
$$

But the $N$ ! permutations of $\mathscr{S}(N)$ define a special subclass of the $2^{N^{2}}$ subsets $M$, of the form $\{(i, p(i)) \mid 1 \leqq i \leqq N\}$.

\section{Appendix C. On the Sums of Squares}

It is a classical result of number theory, that $n \neq 4^{a}(8 m+7), n, a, m$ non-negative integers, is a necessary and sufficient condition for $n$ to be the sum of three nonnegative squares [16]. The maximum number of consecutive integers, which are not representable by three squares, is therefore two: One of them has to be odd and is therefore $8 m+7$, the other must be $8(m+1)$, since $8 m+6$ is not divisible by four. The simplest example is $m=13$, giving $13.8+7$ and $14.8=4^{2} .7$ as consecutive numbers which are not the sum of three squares.

The above consideration solves the question of the biggest gap between consecutive energy levels in a cube with Neumann boundary conditions. For the Dirichlet boundary conditions one has to find the maximum number of consecutive integers, which are not the sum of three strictly positive squares. If $n$ is a sum of two squares, $n+1$ is a sum of three squares, so, the worst situation one can think of, is a sequence $n$ to $n+4$ of the form

$$
8 m+7, \quad 4^{a}(8 k+7), \quad l^{2}, \quad l^{2}+1,
$$

none of them being a sum of three positive squares. It may be, that such a sequence does not really occur, but we have the desired upper bound on the energy gap.

Acknowledgement. This paper would not have been written without many stimulating discussions with $W$. Thirring and $A$. Pflug.

\section{References}

1. Wehrl, A.: On the relation between classical and quantum mechanical entropy. Rep. Math. Phys. (in press.)

2. Thirring, W.: Vorlesungen über mathematische Physik T8: Quanten-Mechanik großer Systeme. University of Vienna lecture notes, to be published in revised form as [15]

3. Lieb, E. H.: Proof of an entropy conjecture of Wehrl. Commun. Math. Phys. 62, 35-41 (1978)

4. Simon, B.: Commun. Math. Phys. 71, 247-276 (1980)

5. Lieb, E.: Commun. Math. Phys. 31, 327-340 (1973)

6. Berezin, F.: Math. USSR 56 88, 269 (1972)

7. Berezin, F.: Math. USSR Izv. 6, 117 (1972) 
8. Golden, S.: Phys. Rev. 137B, 1127-1128 (1965)

9. Thompson, C.: Jour. Math. Phys. 6, 1812-1813 (1965)

10. Beckenbach,E. F., Bellmann, R.: Inequalities. Ergebnisse der Mathematik und ihrer Grenzgebiete, Band 30. Berlin Heidelberg New York: Springer 1971

11. Robinson, D. W.: The thermodynamic pressure in quantum statistical mechanics. Berlin Heidelberg, New York: Springer 1971

12. Marcus, M.: Bull. Am. Math. Soc. 71, 360-364 (1965)

13. Perelomov, A. M.: Coherent states for arbitrary lie group. Commun. Math. Phys. 26, 222-236(1972)

14. Treves, F.: Topological vector spaces, distributions and kernels. New York, London: Academic Press 1967

15. Thirring, W.: Lehrbuch der mathematischen Physik 4. Vienna, New York: Springer 1980

16. Hardy, G. H., Wright, E. M.: An introduction to the theory of numbers. London: Clarendon Press 1954

Communicated by E. Lieb

Received October 23, 1979. 
